\section{Comparative Evaluation of Laser-Based Microarray Scanners}

BioTechniques 31:546-552 (September 2001)

\section{ABSTRACT}

Laboratories use different laser-based scanners to scan microarray images. To assess whether results from different scanners are comparable, and thus whether data from different laboratories can be compared, we scanned the same microarray slide with three commercial scanners that use different imaging techniques. After the acquisition of the microarray images produced by the three scanners, the images were quantified using a single imaging software package and protocol. The results were compared, and we found that the data obtained from the three scanners were comparable and that the variations caused by the use of different instruments were negligible, in spite of the fact that the scanners were based on different optical imaging techniques.

\section{INTRODUCTION}

Microarray is a powerful technique for profiling gene expression in the functional genomics area. This technique has recently been used to shed light into many biological issues, such as disease gene discovery and the molecular classification of cancers (1,5-7). The effective immobilization of DNAs on coated glass offers numerous advantages over membrane binding, such as low background fluorescence of the glass support and the ability to hybridize with dual labeled target probes (3). Glass slides are mechanically arrayed with thousands of DNA spots by means of a robotic system. The generated microarrays are then subjected to co-hybridization assays using fluorescence-labeled target probes prepared from cellular total RNA. Following hybridization, relative expression levels are determined by establishing a ratio of fluorescence intensity between the two labeled targets. The detection of a microarray image is based on the mea- surement of its fluorescence intensities, which allows the simultaneous determination of the relative expression levels of all genes represented on the array. The imaging technique requires light of a specific wavelength to excite the fluorophores in the sample, and scanners can accomplish this by using one of two main techniques. Most scanners use laser sources to excite each fluorescent probe, and emitted light is detected by a photomultiplier tube (PMT). In this first method, a laser-based scanning system involves the rapid movement of small points of laser light across the sample, from which the image is then reconstructed. The second imaging technique is based on a charge-coupled device (CCD). Scanners that use this method have a whitelight arc lamp as their illumination source. Because white light encom passes all wavelengths in the visible spectrum, it is possible to select the wavelength of choice. The fluorescent light emitted from the sample is collected and imaged using CCD cameras.

Cy5 and Cy 3 are two of the most common fluorescent dyes used to label probes. They are excited when the scanner's laser source is set at wavelengths of $633 \mathrm{~nm}$ for Cy 5 and $543 \mathrm{~nm}$ for Cy3. The light emitted is processed through a PMT, and the data collected from each fluorescent channel are stored as an individual 16-bit TIFF file. The images can be scanned at several resolutions, commonly ranging from 5 to $50 \mu \mathrm{m}$.

The whole process of microarray technology involves many steps, which is the reason why many of the current protocols are standardized to minimize experimental variations. However, the reliability of the data from experiment to experiment and the comparison of data from laboratory to laboratory or instrument to instrument have not been extensively evaluated. A commonly accepted standard with which to compare the performances of various scanners is presently lacking.

To evaluate the performances of various scanners, we compared images of the same microarray produced by three different laser-based microarray scanners that relied on different optical mechanisms for image processing. The images were quantified, and the data were analyzed using an ArrayVision ${ }^{\mathrm{TM}}$ software package (Imaging Research, St. Catharines, ON, Canada), rather than the software packages provided with the instruments, to minimize the variability in data processing. An advantage of this software was that it allowed the simultaneous analysis of multiple arrays, from which comparative expressions profiles were established using the quantitative information extracted by the program.

\section{MATERIALS AND METHODS}

Target cDNA spots (4800) were arrayed on a standard glass slide coated with poly-L-lysine. The cDNA was generated by PCR amplification using a Tetrad $^{\mathrm{TM}}$ thermalcycler (MJ Research, Waltham, MA, USA) and purified using MAHV N4550 multiscreen-HV, 96-well filtration plates (Millipore, Bedford, MA, USA). The microarray was generated using a Flexys ${ }^{\circledR}$ arrayer with 48 pins (Genomic Solutions, Ann Arbor, MI, USA). The arrayed slides were UV-cross-linked with $650 \mu \mathrm{J}$ energy. The array was hybridized with fluorescent $\mathrm{Cy} 3$ - and Cy5-labeled total RNA probes in ExpressHyb ${ }^{\mathrm{TM}}$ solution (Clontech Laboratories, Palo Alto, CA, USA) for $16 \mathrm{~h}$ at $42^{\circ} \mathrm{C}$ in a moist chamber. After the hybridization and washing, the slides were scanned using three different scanners: A, B, and C. Scanner $A$ is a four-laser-based imaging system that uses PMT detectors and has a proprietary dark-field illumination to minimize background signal. Scanner $B$ is a simultaneous dual laser scanner with a large field depth of $60 \mu \mathrm{m}$. Scanner $\mathrm{C}$ uses patented confocal laser scanning with the capability to calibrate the PMT. ArrayVision was the image-processing software used to quantify images. Statistica ${ }^{\mathrm{TM}}$ (StatSoft, Tulsa, OK, USA) was used to carry out statistical data analysis.

\section{RESULTS AND DISCUSSION}

Table 1 briefly summarizes the com parison of technical specifications of the three instruments. Scanner A, which was a sequential scanner (scans one channel after another), combined dark-field imaging and laser-beam 


\section{Short Technical Reports}

Table 1. Comparison of the Specifications of the Three Scanners

\begin{tabular}{|llll|}
\hline Specifications & Scanner A & Scanner B & Scanner C \\
\hline Imaging & Dark-Field & Simultaneous & Confocal \\
Field Focus $(\mu \mathrm{m})$ & "unknown" & 60 (thick specimen) & 10 (small depth) \\
$\begin{array}{c}\text { Scan Resolution } \\
(\mu \mathrm{m})\end{array}$ & 10,20 , and 50 & 10 and 40 & $5,10,20$, and 50 \\
$\begin{array}{l}\text { Scanning } \\
\text { Parameters }\end{array}$ & PMT adjustment & PMT adjustment & PMT and laser \\
File Format & TIFF & TIFF & TIFF \\
Dimensions (In.) & $36 \times 24 \times 22$ & $12 \times 18 \times 7$ & $30 \times 16 \times 14$ \\
\hline
\end{tabular}

scanning with PMT detection. A darkfield approach angles the laser beam so that the back-scattered laser beam is prevented from reaching the PMT. Consequently, the only photons to reach the detector are those emitted by the cDNA molecules, rendering this particular technique extremely useful in terms of the high contrast and improved signal it provides. Likewise, dark-field imaging improves both the sensitivity and the dynamic range of the instrument. As a drawback, this technology yields images that are dimmer than those from scanners using other techniques and thus requires longer expo- sure to obtain a good signal. In the process, there is a greater level of photobleaching of the fluorescent signal.

Scanner B carried out the simultaneous imaging of the two fluorescent probes; hence, its scanning process was fast. However, this increased speed was achieved at the expense of an enhancement of both signal and background. Likewise, although simultaneous imaging avoids pixel shifting, a large data file of $64 \mathrm{MB}$ is generated. With this scanner, a full-power laser beam that scans across the entire slide is used. The fluorescence of $\mathrm{Cy} 3$ and $\mathrm{Cy} 5$ are measured at 532 and $635 \mathrm{~nm}$, respectively. Although the emission maximum of Cy3 is $543 \mathrm{~nm}$, a lower wavelength is chosen to achieve a better spectral separation of the two fluorophores and to reduce any potential cross-talk during the simultaneous scanning process. If other fluorescent probes are used, their emission wave- 


\section{Short Technical Reports}

A
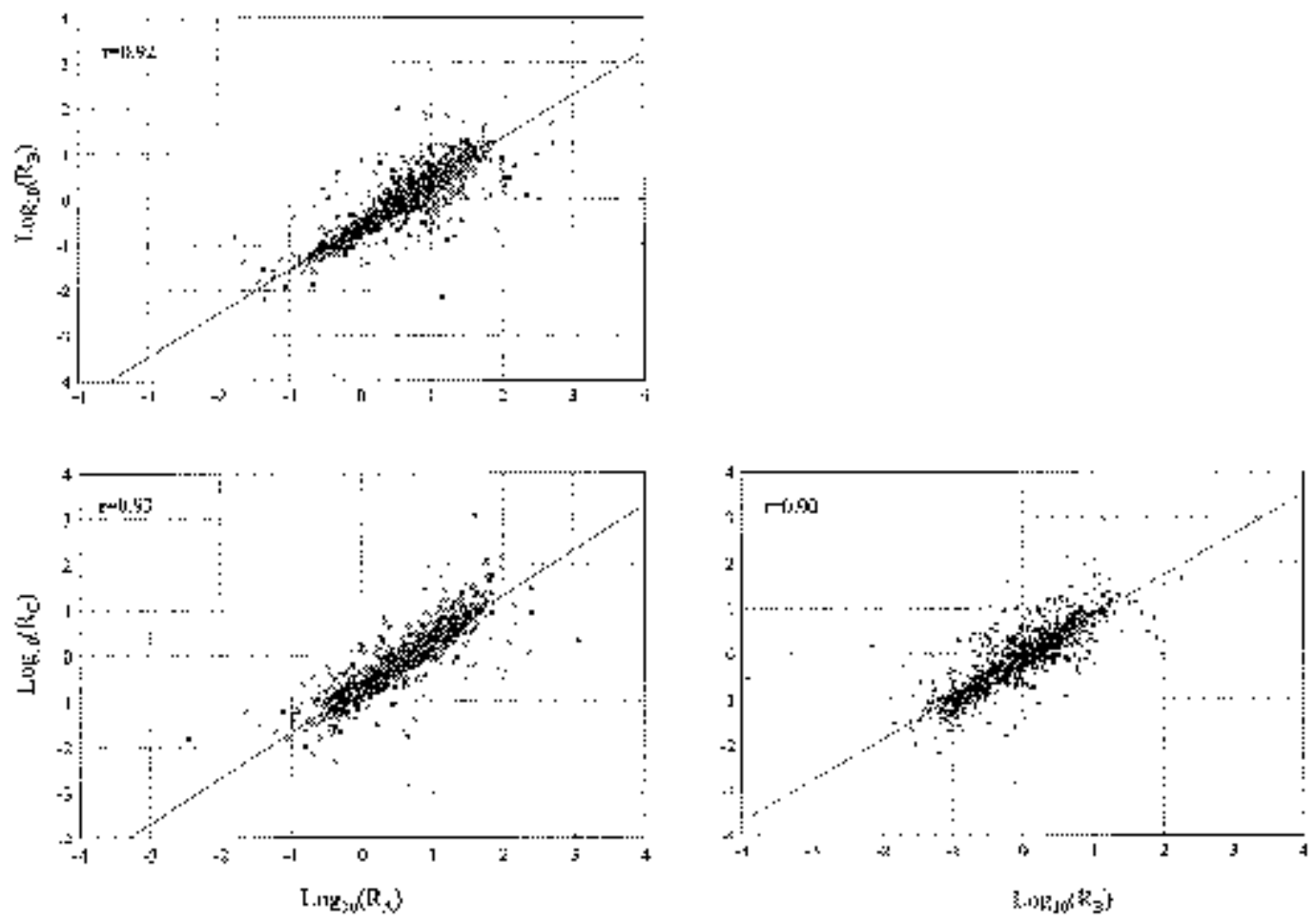

B
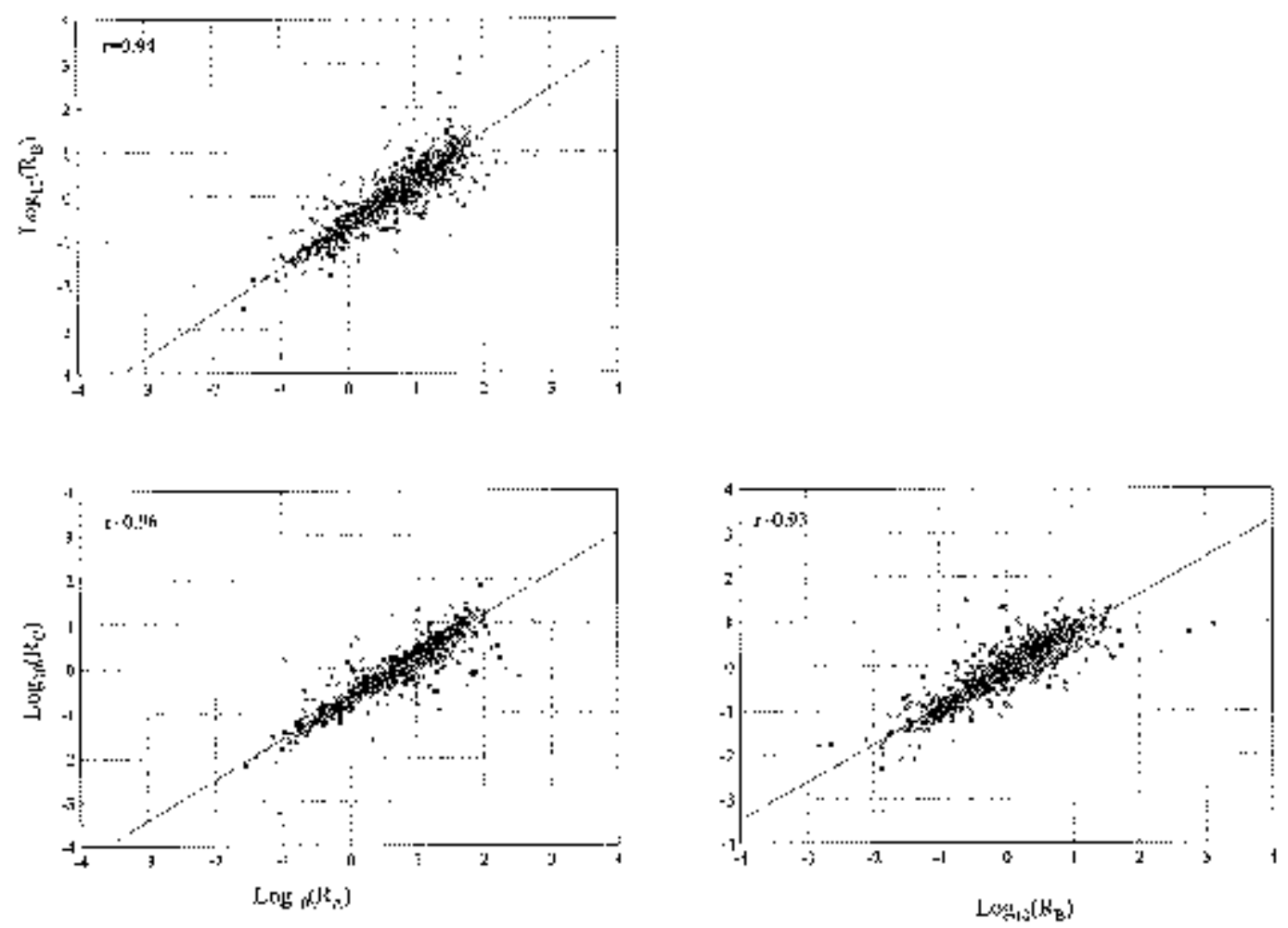

Figure 1. Correlation analysis among three scanners. (A) $20-\mu \mathrm{m}$ and (B) $10-\mu \mathrm{m}$ resolution. The correlation coefficients (r) are labeled in each of the com parisons. $R_{A}, R_{B}$, and $R_{C}$ in the figure axis represent data obtained from scanners $A, B$, and $C$, respectively. $\log _{10}(R)$ is the logarithm-transformed intensity ratios of the two channels. A linear regression line is shown in each plot. 


\section{Short Technical Reports}

lengths also have to be separated enough to avoid cross-talk.

Scanner C used confocal imaging technology. With this type of imaging, the laser source is focused to a point on the sample to be imaged at a depth of $10 \mu \mathrm{m}$. An upstream detector allows the detection of photons emitted from the area on the sample upon which the pinhole is focused. In this manner, only the "in focus" light (from the microarray spots) makes it into the detector, rendering a presumably high signal-tonoise ratio. The scanning procedure is single-pass.

Based on an understanding of the different imaging techniques used by the three scanners, we first expected to find differences in their quantification of data. Instead of using the acquisition and analysis software provided by each manufacturer of the instruments, the 16-bit image files were retrieved by ArrayVision as TIFF files and analyzed after setting up the array's alignment parameters. The protocol selected for analysis provided: ( $i$ ) measurements of volumes, defined as the density of each spot multiplied by its area; (ii) background (background volume), defined as the corners between spots, with the size of each background spot being four pixels; (iii) sVol, defined as the background corrected volume, (i.e., the volume of the spot minus the background volume); and (iv) ratio, defined as the data from each spot in one channel image divided by the data from the corresponding spot in another channel.

The ratio of sVols from two channels (Cy3 and Cy5) was used as the relative expression level (R). Because the two sets of quantitative data from each of the channels varied over a large range, we carried out the comparison and correlation analyses after transforming the data into a logarithmic value $(2,4)$.

A detailed comparison of data pairs was carried out using logarithm-transformed intensity ratios, defined as

$\log _{10}\left(\frac{R_{1}}{R_{2}}\right)$

where

$R_{1}=\left(\frac{C y 5_{\text {sVol }}}{C y 3_{\text {sVol }}}\right)$ Instrument 1 and $R_{2}=\left(\frac{C y 5_{\text {sVol }}}{C y 3_{\text {sVol }}}\right)_{\text {Instrument } 2}$

[Eq. 2]
Figure 1, A and B, presents the com parisons between the logarithm 10 -based ratios for all three scanners. The array data obtained from a low-resolution (20 $\mu \mathrm{m})$ scan are illustrated in Figure 1A, while Figure $1 \mathrm{~B}$ reflects the data obtained from a $10-\mu \mathrm{m}$ resolution scan. High correlation coefficients ( $r$ ) between each pair of scanners implied that the data produced by the different scanners were in agreement. The data obtained at the higher resolution $(10 \mu \mathrm{m})$ were better correlated than data obtained at the lower resolution $(20 \mu \mathrm{m})$. The correlation coefficient for a channel-to-channel comparison between the instruments was greater than 0.95 .

It is worth noting that when images and data were obtained using the same scanner at different times, the correlation coefficient was about 0.93 . This number fell within the range of variation observed for the data obtained from the different scanners $(r=0.90-$ 0.96). Thus, any variability observed was not a result of the instrument used.

We also compared the data analysis of the differentially expressed genes of the two co-hybridized samples acquired from the three scanners. We used a threshold ratio of greater than 3.0. Among the top-ranked 160 genes, there was $95 \%$ agreement with the genes from the three analyses. Thus, the three instruments are identifying the same genes as differentially expressed.

Although the three commonly used laser scanners differed in a number of technical features, the gene expression data obtained from them were very similar. A practical consequence of this observation is that data produced by different laser scanners from various laboratories can be reliably compared and analyzed.

\section{REFERENCES}

1.Bittner, M., Y. Meltzer, Y. Chen, Y. Jiang, E. Seftor, M. Hendrix, M. Radmacher, R. Simon et al. 2000. Molecular classification of cutaneous malignant melanoma by gene expression profiling. Nature 406:536-540.

2.Chen, Y., E.R. Dougherty, and M.L. Bittner. 1997. Ratio-based decisions and the quantitative analysis of cDNA microarray images. J. Biomed. Opt. 24:364-374.

3.Cheung, V.G., M. Morley, F. Aguilar, A. Massimi, R. Kucherlapati, and G. Childs. 1999. Making and reading of microarrays.
Nat. Genet. 21:15-19.

4.Dudiot, S., Y.H. Yang, M.J. Callow, and T.P. Speed. Statistical methods for identifying differentially expressed genes in replicated cDNA microarray experiments. J. Am. Stat. Assoc. (In Press).

5.Eisen, M.B. and P.O. Brown. 1999. DNA arrays for analysis of gene expression. Methods Enzymol. 303:179-205.

6.Fuller, G.N., C.H. Rhee, K. Hess, L. Caskey, R. Wang, J.M. Bruner, W.K.A. Yung, and W. Zhang. 1999. Reactivation of insulin-like growth factor binding protein 2 expression in glioblastoma multiforme: a revelation by parallel gene expression profiling. Cancer Res. 59:4228-4332.

7.Hegde, P., R. Qi, K. Abernathy, C. Gay, S. Dharap, R. Gaspard, J.E. Hughes, E. Snesrud et al. 2000. A concise guide to cDNA micorarray analysis. BioTechniques 29:548-562.

This work was partially supported by a donation from the Michael and Betty $\mathrm{Ka}$ doorie Foundation, the "Tobacco Settlement Funds as appropriated by the Texas State Legislature”, and grant no. 0036570039-1999 from the Texas Higher Education Coordination Board. The authors thank Pilar Laborde for her valuable help with the editorial aspect of the manuscript. Address correspondence to Dr. Wei Zhang, Director, Cancer Genomics Core Laboratory, Department of Pathology, Box 85, The University of Texas M.D. Anderson Cancer Center, Houston, TX 77030, USA. e-mail: wzhang @mdanderson.org

Received 22 November 2000; accepted 11 April 2001.

\section{Ramdas, J. Wang, L. Hu, D. Cogdell, E. Taylor, and W. Zhang \\ The University of Texas}

M.D. Anderson Cancer Center Houston, TX, USA

For reprints of this or any other article, contact Reprints@BioTechniques.com 